Case Report

\title{
UMBILICAL METASTASIS MIMICKING SYMPTOMATIC UMBILICAL HERNIA - A RARE CASE REPORT AND REVIEW OF THE LITERATURE
}

\author{
Iv. Novakov* \\ Department of Special Surgery, Medical University - Plovdiv, Bulgaria
}

\begin{abstract}
Umbilical metastases, also termed Sister Mary Joseph's nodule, are reported rarely in medical literature and they are associated with advanced metastatic intra-abdominal and pelvic cancers. The purpose of this publication is to report a rare case of umbilical metastasis misdiagnosed as acomplicated umbilical hernia.

Case presentation. A 74-years-old woman with nausea and painful umbilical swelling was misdiagnosed as incarcerated umbilical hernia on the initial examination. Abdominal computed tomography revealed a huge pelvic cystic-solid mass, probably originates from right ovary.

Conclusion. In conclusion, the presented case demonstrated that due to the lack of understanding Sister Mary Joseph's nodule could be easily mistaken for incarcerated umbilical hernia.
\end{abstract}

Key words: Sister Mary Joseph's nodule, ovarian cancer, incarcerated umbilical hernia.

\section{INTRODUCTION}

Umbilical metastases also termed Sister Mary Joseph's nodules are reported rarely in medical literature. In general, they are associated with advanced metastatic intra-abdominal and pelvic cancers and indicate poor prognosis (1-6)

Umbilical metastases can be symptom of malignant disease progression or sign of cancer recurrence in a known patient. They also may occur as the presenting sign of undiagnosed malignancy. With a lack of understanding, umbilical metastases could be easily mistaken for omphalitis, cysts, umbilical hernias and skin diseases $(1,2,4)$. The aim of this publication is to report a rare case of umbilical metastasis as the first sign of advanced pelvic cancer, misdiagnosing as incarcerated umbilical hernia on the initial patient's examination.

\section{CASE PRESENTATION}

A 74-years-old woman with nausea and painful umbilical swelling was examined in the emergency department of University hospital.

A painless mass in the umbilicus was accidentally palpated by woman two months before.

Correspondence to: Ivan P. Novakov, Department of Special Surgery, Medical University -Plovdiv, Bulgaria, e-mail: inovakov2003@yahoo.com, mobile phone: +359887575487
Physical examination of the woman revealed a firm, painful, bluish-violet, non-reducible periumbilical mass of approximately $4 \mathrm{~cm}$ in diameter (Figure 1). On inspection the abdomen was moderately distended. On palpation there was moderate diffuse abdominal pain without guarding, and on auscultation increased peristalsis was heard.

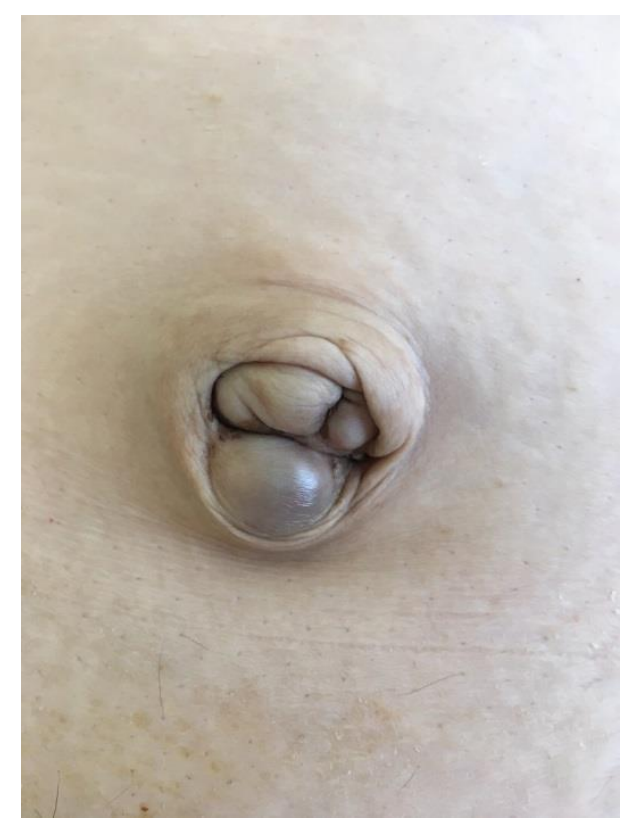

Figure 1. Umbilical swelling with bluish-violet colorization of the skin, initially accepted as a complicated umbilical hernia. 
With diagnosis "incarcerated umbilical hernia" the woman was admitted to the Department of Special Surgery. Due to the suspicion of the diagnosis of admission (based on the new physical examination in the department), computed tomography of the abdomen was performed. It revealed a huge pelvic cysticsolid mass (max. diameter of $17 \mathrm{~cm}$ ), with
NOVAKOVIV.

involvement of uterus, right ovary, and terminal ileum and ascending colon (Figure 2). The computed tomography also demonstrated a solid mass, with maximum diameter of $5 \mathrm{~cm}$, invading the full thickness of the abdomen wall at the umbilical region (Figure 3).

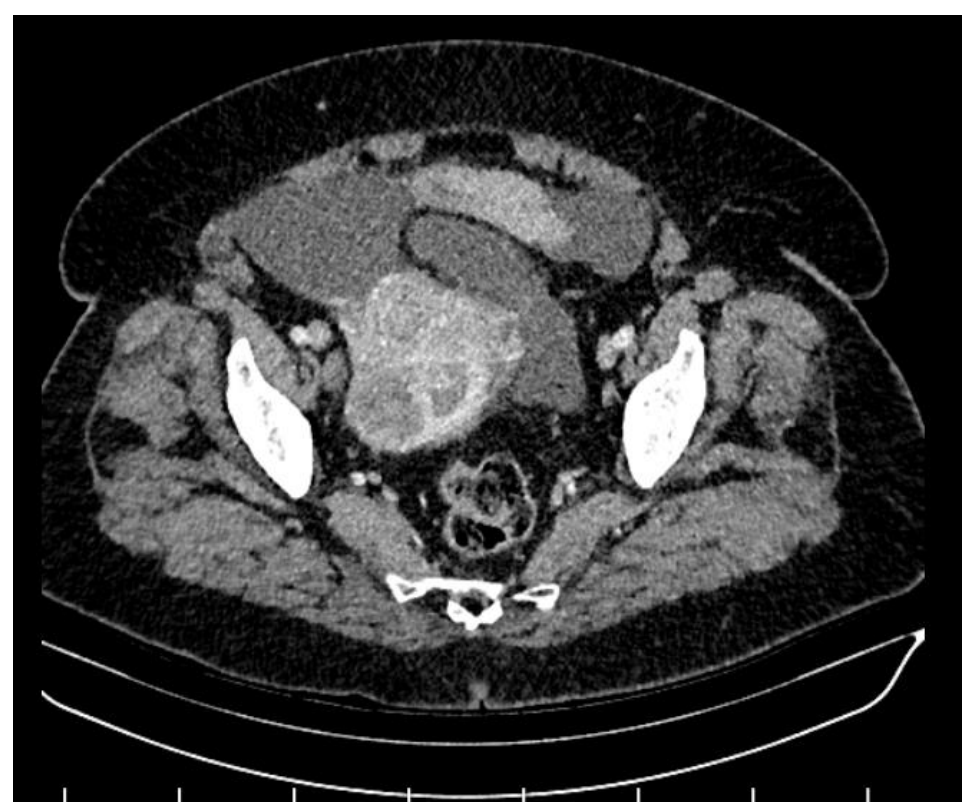

Figure 2. Abdominal computed tomography scan: a large pelvic mass, probably originating from the right ovary.

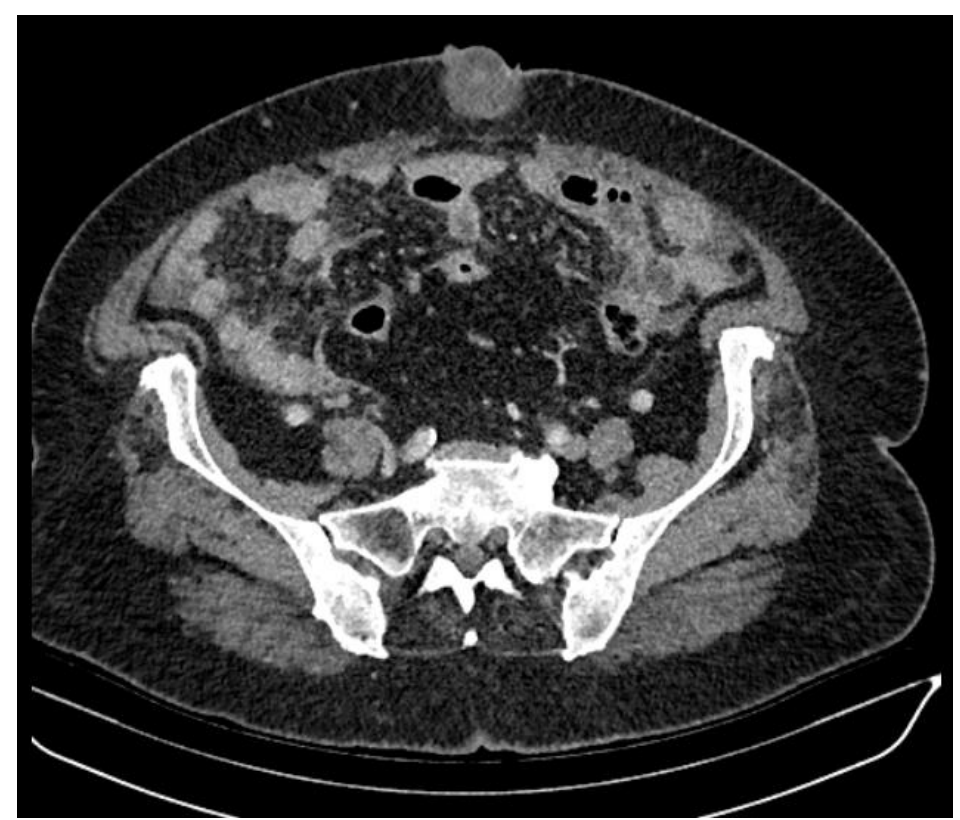

Figure 3. Abdominal computed tomography scan: solid mass at the umbilical region.

The woman underwent a laparotomy (24 hours after the admission). Exploration of the peritoneal cavity established a huge cysticsolid mass of the right ovary with massive infiltration to the parietal pelvic peritoneum, uterus, terminal ileum, coecum and ascending colon. Umbilical metastasis, miliary parietal peritoneal metastases and peritoneal effusion (positive of tumor cells, determined by express cytological examination) were also established. The woman underwent right hemicolectomy, permanent right-sided terminal ileostomy, excision of umbilical metastatic lesion, biopsy 


\section{NOVAKOVIV.}

of ovarian tumor mass and peritoneal metastases.

The final pathology report revealed a cystadenocarcinoma of the right ovary with invasion to the right colon and terminal ileum and distant peritoneal metastases with involvement of the umbilicus. The woman received 6 cycles of chemotherapy over a period of 3 months and after 13 months of follow-up (from initial diagnosis to the preparing of this manuscript) she has been alive.

\section{DISCUSSION}

Metastatic cancer of the umbilicus, known as Sister Mary Joseph's nodule, is an uncommon manifestation of visceral and other malignancies (encountered in about $1-3 \%$ of patients with an intra-abdominal and/or pelvic malignancy). Gastric carcinoma is the most common cause of Sister Mary Joseph's nodule in men, and ovarian carcinoma is the most common cause in women (confirmed by the presenting case) (4-6).

Umbilical metastases may be the only symptom in patients with internal malignancies, and may represent as a late finding in patients with widespread metastases. In generally, the nodule is firm, painless, and irregular in shape. The overlying skin may be white, bluish-violet (like the presented case), and brownish-red, often fissured and ulcerated with a bloody, mucinous, serous, or purulent discharge $(7,8)$.

With a lack of understanding, Sister Mary Joseph's nodule could be easily mistaken for omphalitis, cyst, umbilical hernia and skin disease. Other diseases can be considered among diagnostic hypothesis, such as sarcoma, endometriosis, lymphangioma, lipoma, umbilical granuloma. The presenting case is an interesting example of a difficult initial diagnosis of Sister Mary Joseph's nodule. The complaints of the woman and her physical abdominal finding, initially wrongly determined as the result of incarcerated umbilical hernia, were expression of incomplete bowel obstruction, caused by massive intestinal infiltration by the advanced ovarian cancer $(1-3,9,10)$.

The presence of Sister Joseph's nodule usually signifies advanced, metastasizing cancer and almost certainly means the inoperability of the patient. Even though, the surgery is recommended only in patients with a solitary umbilical metastasis, just like in the presentied case. However, the aim of the surgery was not to treat ovarian cancer. The operation was directed to the intestinal obstruction. The abdominal exploration confirmed the state of the woman - advanced ovarian cancer with intestinal obstruction, and the histological examination of the resected umbilical mass verified Sister Joseph's nodule $(10-14)$.

The presence of an umbilical metastasis usually indicates a poor prognosis - the survival of these patients without treatment has been reported to range from 2 to 11 months from the time of initial diagnosis. There are reports that suggested better prognosis with aggressive chemotherapy in cases of ovarian cancer with umbilical metastasis (the mean survival of 17.6-21 months), which is additionally confirmed by the presented case.

\section{CONCLUSION}

In conclusion, this publication presents one of uncommon manifestation of advanced ovarian carcinoma - umbilical metastasis, known as Sister Mary Joseph's nodule. The presented case demonstrated that due to the lack of understanding Sister Mary Joseph's nodule could be easily mistaken for incarcerated umbilical hernia.

\section{REFERENCES}

1. Varga-Szabó D, Papadakis M, Pröpper S, Zirngibl H. Primary peritoneal adenocarcinoma as content of an incarceratedumbilical hernia: A case-report and review of the literature. International Journal of Surgery Case Reports 2016;19:134-136.

2. Cestaro G, De Monti M, Alkayyali S, Fasolini F, Salmoiraghi F. Umbilical metastasis mimicking symptomatic hernia: Report of a caseof Sister Mary Joseph Syndrome. International Journal of Surgery Case Reports 2017;41:105-106.

3. Jian-Syun Chen, Chien-Kuo Liu. Sister Mary Joseph's Nodule: Ascending Colon Cancer with Umbilical Metastasis. J. Cancer Res. Pract. 2015;2:319-324.

4. Yong You Wu, Chun Gen Xing, Ju Xiang. Carcinoma of the right side colon accompanied by Sister Mary Joseph's nodule and inguinal nodal metastases: a case report and literature review. Chinese Journal of Cancer 2010;29:239-242. 
5. Gabriele R, Conte M, Egidi F. Umbilical metastases: current viewpoint. World $J$ Surg Oncol, 2005;3:13-16.

6. Zhigang Zhang, Jianwei Wang, Jian Huang. Umbilical metastasis derived from early stage rectal cancer: a case report. World Journal of Surgical Oncology 2014;12:8286.

7. Larentzakis A, Theodorou D, Klio Fili. Sister Mary Joseph's nodule: Three case reports. Cases Journal 2008;1:182-185.

8. M. Palaniappan, W.M. Jose, A. Mehta. Umbilical metastasis: a case series of four Sister Joseph nodules from four different visceral malignancies. Current Oncology 2010;17:78-81.

9. Urbano F. Sister Joseph's Nodule. Review of Clinical Signs 2001;44:33-35.

\section{NOVAKOVIV.}

10. Piura B, Meirovitz M, Bayne M, ShacoLevy R. Sister Mary Joseph's nodule originating from endometrial carcinoma incidentally detected during surgery for an umbilical hernia: a case report. Arch Gynecol Obstet 2006; 274:385-388.

11. J.R. Benson, S. Singh. Joseph's nodule: a case report and review. Eur. J. Surg.Oncol. 1997;23:451-454.

12. Piura B, Umbilical metastasis: Sister Mary Joseph's nodule. Harefuah 2006;7:505-509.

13. Aich RK, Karim R, Chakraborty B. Sister Mary Joseph's nodule. Indian $\mathrm{J}$ Med Paediatr Oncol 2008;2:40-43.

14. Dar IH, Kamili MA, Dar SH. Mary Joseph nodule-A case report with review of literature. J Res Med Sci 2009;14:385-387. 